\title{
A new approach to geographic routing for location aided cluster based MANETs
}

\author{
SenthilVelmurugan Mangai ${ }^{{ }^{*}}$ and Angamuthu Tamilarasi ${ }^{2}$
}

\begin{abstract}
Routing has been the main challenge for ad hoc networks due to dynamic topology as well as resource constraints. Completely GPS(Global Positioning System) free as well as GPS scarce positioning systems for wireless, mobile, ad-hoc networks has been proposed recently by many authors. High computational overhead and high mobility of the nodes typically require completely GPS enabled MANETs for higher performance. In this article, Improved Location aided Cluster based Routing Protocol (ILCRP) for GPS enabled MANETs has been evaluated for performance metrics such as end to end delay, control overhead, and packet delivery ratio. Use of cluster based routing as well as exact location information of the nodes in ILCRP reduces the control overhead resulting in higher packet delivery ratio. GPS utility in nodes reduces the end to end delay even during its high mobility. Simulations are performed using NS2 by varying the mobility (speed) of nodes as well as number of the nodes. The results illustrate that ILCRP performs better compared to other protocols.
\end{abstract}

Keywords: MANET, GPS, Routing Algorithm, Location aided routing, Cluster based routing, Stable clustering

\section{Introduction}

'Resource Constraint' is an extreme challenge faced by a routing protocol designed for ad hoc wireless networks. Gadgets used in the ad hoc wireless networks in most cases require portability and hence they also have size and weight constraints along with the restrictions on the power source. Control overhead increases due to mobility of the nodes resulting in bandwidth constraint. Mobility also affects end to end delay as well as packet delivery ratio. Therefore, in real time applications there is a reduction in quality due to bandwidth constraint. As a result, ad hoc network routing protocols must optimally balance these contradictory aspects.

Many routing protocols [1] have been proposed to reduce the complexity of a flat structured routing either with help of the clustering schemes or using location information of the nodes. Through clustering, MANETs are partitioned into a group of nodes with a Cluster Head $(\mathrm{CH})$. These clusters are dynamically rearranged with change in topology of the network. $\mathrm{CH}$ is the node which represents itself as a single entity and has specific

\footnotetext{
* Correspondence: ishamangai@yahoo.com

'Department of Electronics \& Communication Engineering, Velalar College of Engineering and Technology, Thindal, Erode-638 012, Tamil Nadu, India Full list of author information is available at the end of the article
}

responsibilities. Cluster members are simply nodes that join a cluster but cluster members that belong to more than one cluster are gateway nodes. The gateway nodes are used for communication between clusters. When there is more than one gateway to the same cluster, the $\mathrm{CH}$ chooses the best one for routing data by considering the node value of each gateway node. If two clusters are non-overlapping then each cluster will have separate gateway nodes. These gateway nodes will facilitate inter $\mathrm{CH}$ communication.

\section{Related work}

Many algorithms have been proposed to optimize the procedure for election of $\mathrm{CH}$. Lowest-ID algorithm [2,3] uses minimum ID whereas Highest-Degree (HD) [4] uses degree of the node as a metric for $\mathrm{CH}$ election. The degree of a node is the number of neighbour nodes. LID biases the lower ID to drain their resource ultimately leading to node failure. Even though HD reduces the delay as well as the number of clusters, it increases reaffliation overhead resulting in higher number of re-elections.

Mobility Metric Based Algorithm (MOBIC) [5], a variation of Lowest-ID algorithm, uses the ratio of two consecutive signal strengths received by a node to know its

\section{SpringerOpen $^{\odot}$}

(c) 2011 Velmurugan and Angamuthu; licensee Springer. This is an Open Access article distributed under the terms of the Creative Commons Attribution License (http://creativecommons.org/licenses/by/2.0), which permits unrestricted use, distribution, and reproduction in any medium, provided the original work is properly cited. 
relative motion with respect to its neighbors. MOBIC applies well only for group mobility of the nodes. MOBIC provides stability at the cost of higher delay and can be applicable only to group mobility of the nodes.

Node mobility as well as transmission range are taken for weight calculation in Distributed Mobility Adaptive Algorithm (DMAC) [6]. Most of the algorithms such as Weighted Clustering Algorithm (WCA) [7-9], Generalized Distributed Mobility Adaptive Clustering (GDMAC) [10] are derived from DMAC. WCA considers degree of connectivity, mobility, battery power and transmission power. WCA is extended to improve performances in IWCA [11], FWCA [12]. GDMAC improves the performance by introducing a cluster density parameter for the whole network. WCA and its derived algorithms provide better performance with compromised setup delay. Introduction of more parameters result in setup delay.

Similarly, many weighted algorithms are proposed for electing a $\mathrm{CH}$. Apart from algorithms, protocols such as CEDAR, CBRP, etc. improve the scalability as well as performance of MANETs.

Cluster Based Routing Protocol (CBRP) $[13,14]$, an on demand source routing protocol, divides clusters into nodes and decreases control overhead during route discovery. K-Hop CBRP [15] improves CBRP [14] with increase in number of nodes and its mobility. It modifies the existing WCA for the election of $\mathrm{CH}$.

In Location Aided Routing (LAR) [16] protocol the overhead of route discovery is decreased by utilizing location information of mobile nodes. Using GPS [17] for location information, LAR protocol reduces the search space for a desired route. Reducing the search space results in fewer route discovery messages. By contacting a location service provider which knows the positions of all the nodes, the source node should first get the position of the destination mobile node when it wants to send data packets to a destination.

To localize the ad hoc network, a wide variety of routing protocols [18-20] have been proposed over the years. Some techniques use GPS but for very few nodes. These nodes are often referred to as anchor nodes or reference nodes. 'Completely GPS Free Localization' [21-24] or 'Using Very Few Anchor Node' $[25,26]$ are the two types of localization approaches that provide techniques to localize the network in a GPS Less or GPS-Scarce area (LACBER). The GPS-less localization [27] approaches, establish a virtual coordinate system and try to localize the network in that coordinate system. On the basis of distance measurement (using ToA or AoA or RSSI) or hop count these coordinate systems are established. Using the above coordinate systems, the exact location of the node cannot be determined due to absence of GPS.
Location Aided Cluster Based Energy-efficient Routing (LACBER) [28] is a location aided routing protocol proposed for GPS scarce ad hoc networks. In the network, only a few nodes are GPS enabled and are capable of finding their own location using GPS. A few special nodes are equipped with antennas which can measure RSSI and the angle of arrival (AOA) of received signals from other nodes. The rest of the network can find their positions in a process using either GPS enabled or special nodes.

The LACBER protocol requires that each cluster must have at least one GPS enabled node or antenna equipped node in it. Compared to other cluster based routing protocols [29] the formation of clusters in LACBER protocol results in high control overhead. Using LACBER protocol, determining the location of normal nodes with high mobility is a constraint.

\section{Proposed protocol}

This article proposes an ILCRP protocol where all the nodes in all the clusters are GPS enabled compared to few nodes in a cluster as in LACBER protocol. The proposed protocol makes use of clusters as well as location information intensively. The exact location information of the nodes is known to each other with the help of GPS. The protocol is divided into three phases. First phase is cluster formation followed by cluster maintenance. The last phase is route discovery phase.

In the proposed ILCRP protocol, the control overhead becomes less for route discovery due to its GPS capability. The proposed protocol delivers the packets more accurately with less end to end delays since the exact location of the source as well as destination nodes are known to respective CHs. Besides, the overhead decreases due to exact location information of the nodes at all $\mathrm{CHs}$.

\section{Cluster formation}

Clusters are formed between nodes which are m-hops far away from the $\mathrm{CH}$. All the nodes start in undecided stage. Since all the nodes are GPS enabled, all the nodes can become $\mathrm{CH}$. Initially all the nodes in the network broadcast a HELLO (Table 1) message with node ID and location information. Location information is obtained using GPS utility with an assumption of location error e. Let node ID be the MAC address as stated in FWCA. Based upon the updated neighbour nodes' list, the node calculates its Node Value. Each node computes its node value based on the following parameters:

- The degree difference $\Delta \mathrm{i}$ : It is defined as the difference between the cluster's size ' $N$ ' and the actual number of neighbors. It allows estimating the remaining number of nodes that each node can still handle. 
Table 1 Selection of cluster head

\begin{tabular}{|c|c|c|c|c|c|c|}
\hline $\begin{array}{c}\text { No. of nodes } \\
\mathrm{N}_{i} \text { in the cluster } C_{i}\end{array}$ & $\begin{array}{c}\text { Weights } \\
W_{1}, W_{2}, W_{3}\end{array}$ & $\begin{array}{l}\text { Degree } \\
\text { difference }\end{array}$ & $\begin{array}{c}\text { Mobility } \\
M \\
\text { in } \mathrm{m} / \mathrm{s}\end{array}$ & $\begin{array}{l}\text { Remaining battery power } \\
\text { in J }\end{array}$ & $\begin{array}{l}\text { Node value } \\
\text { NV }\end{array}$ & $\begin{array}{l}\text { Selected node as cluster } \\
\text { head }\end{array}$ \\
\hline $\begin{array}{c}3 \\
\left(N_{1}, N_{2}, N_{3}\right) \\
\end{array}$ & $\begin{array}{l}(0.09,0.38 \\
0.53)\end{array}$ & $7,5,2$ & $2,4,6$ & $200,150,150$ & $106,78,77$ & $N_{1}$ \\
\hline $\begin{array}{c}5 \\
\left(\mathrm{~N}_{4}, \mathrm{~N}_{5}, \mathrm{~N}_{6}, \mathrm{~N}_{7}, \mathrm{~N}_{8}\right) \\
\end{array}$ & $\begin{array}{c}(0.27,0.31 \\
0.42) \\
\end{array}$ & $2,6,4,8,5$ & $3,1,3,1,7$ & $\begin{array}{c}174,190,188 \\
200,182 \\
\end{array}$ & $73,81,79,86,76$ & $\mathrm{~N}_{7}$ \\
\hline $\begin{array}{c}6 \\
\left(N_{9}, N_{10}, N_{11}, N_{12}, N_{13},\right. \\
\left.N_{14}\right)\end{array}$ & $\begin{array}{l}(0.33,0.24 \\
0.43)\end{array}$ & $3,4,9,8,7,2$ & $2,3,1,5,4,2$ & $130,156,195,169,179,120$ & $\begin{array}{l}56,68,87 \\
74,78,52\end{array}$ & $N_{11}$ \\
\hline
\end{tabular}

$\Delta \mathrm{i}=|\mathrm{di}-\mathrm{N}|$ where di is the degree of the node and $N$ is the threshold for number of nodes in the cluster

- The mobility of the node M.

Mobility of the node at time $t_{2}$ is calculated using the below formula:

$$
M=\frac{1}{\left(t_{2}-t_{1}\right)}\left(\sqrt{\left(x_{2}-x_{1}\right)^{2}+\left(y_{2}-y_{1}\right)^{2}}\right)
$$

Where $\mathrm{x}_{1}, \mathrm{y}_{1}$ and $\mathrm{x}_{2}, \mathrm{y}_{2}$ are the co ordinates of the node at time $T_{1}$ and $T_{2}$ respectively.

- The remaining battery power of the node is $\mathrm{P}_{\mathrm{a}}$.

Therefore, Node Value $\mathrm{NV}=\mathrm{W}_{1} \times \Delta \mathrm{i}-\mathrm{W}_{2} \times \mathrm{M}+\mathrm{W}_{3}$ $\times \mathrm{P}_{\mathrm{a}}$ where $\mathrm{W}_{1}, \mathrm{~W}_{2}, \mathrm{~W}_{3}$ are the weights used and are in a relation such that $\mathrm{W}_{1}+\mathrm{W}_{2}+\mathrm{W}_{3}=1$. Maximum node value of a Node can be calculated by considering the mobility of the node as NULL. The threshold value is the value till which the elected $\mathrm{CH}$ retains the head of the cluster and is approximately given by forty percent of the maximum node value.

All the nodes, after finding its node value NV, broadcasts NV using an INFO (Table 1) message to its 1-hop neighbors. Depending upon the node values, the node with the highest node value and greater than the threshold value of the maximum node value elects itself as $\mathrm{CH}$ by sending $\mathrm{CH}_{-}$INFO. Table 1 shows the method of selection of the $\mathrm{CH}$ for three clusters.

CH_INFO (Table 2) is the packet broadcasted by $\mathrm{CH}$ on its self election as $\mathrm{CH}$ containing its ID and the neighbor table. Neighbor table is a conceptual data structure for formation of a cluster whereas Cluster Adjacency Table (CAT) is used for keeping information about the adjacent clusters. In CAT, CH stores the IDs of the adjacent $\mathrm{CHs}$, gateway node IDs to reach adjacent $\mathrm{CHs}$, whereas nodes store NULL. Gateway node is the node through which the $\mathrm{CH}$ communicates with an adjacent cluster. Neighbor Table is used for intra cluster routing and CAT is for inter cluster routing. Adjacency cluster discovery and gateway node selection are done as per the CBRP IETF MANET draft. All other nodes store node IDs, location information and its node values in its neighbor tables. In Figure 1, the cluster $\mathrm{C} 1$ has one $\mathrm{CH}$, one gateway node and four member nodes.

\section{Cluster maintenance}

The clusters have to be reorganized and reconfigured dynamically due to the mobility of nodes in the ad hoc network. There are three major scenarios in a cluster for reconfiguration. The scenarios are:

- Reduction in the node value of the $\mathrm{CH}$

- Mobility of a node

- Mobility of $\mathrm{CH}$

\section{Reduction in the Node Value of the Cluster Head}

The $\mathrm{CH}$ determines its node value from time to time. When its node value falls below threshold value, the $\mathrm{CH}$ sends CH_RELEIVE (Table 2) to all its nodes in its cluster. After receiving CH_RELEIVE, all the nodes calculate the respective node values and convey them to the $\mathrm{CH}$.

Table 2 Summarizes the messages used for formation as well as maintenance of the clusters

\begin{tabular}{cr}
\hline Message & Description \\
\hline HELLO & Contains broadcaster's ID, location information, node status, neighbour table, cluster adjacency table and sender's node value \\
\hline INFO & Contains node value \\
\hline CH_INFO & Contains cluster head ID and cluster neighbour table \\
\hline CH_ACK & The new node's HELLO message is acknowledged by cluster head (CH) \\
\hline JOIN & A new node joins as member in the cluster after cluster head (CH) is activated by sending JOIN message \\
\hline CH_NEWNODE & The new node's JOIN is acknowledged by cluster head. \\
\hline CH_NACK & The new node's HELLO is rejected by cluster head \\
\hline CH_RELIEVE & Notifies the members about its intention to resign as cluster head \\
\hline CH_RACK & Present cluster head relieves finally after broadcasting new cluster head ID
\end{tabular}




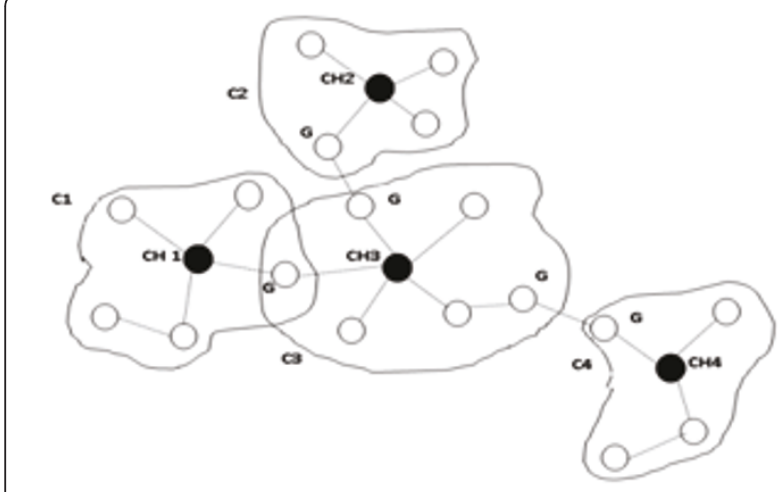

Figure 1 ILCRP cluster formation.

Now the $\mathrm{CH}$ decides the next succeeding $\mathrm{CH}$ with CH_RACK (Table 2) with node ID of the new $\mathrm{CH}$.

\section{Mobility of a node}

When a node goes from one cluster to another, the state becomes undecided and it floods the new network with HELLO message containing important information regarding the sender such as sender's ID, location information, node status, neighbour table, CAT and its node value. On receiving the HELLO message, the $\mathrm{CH}$ verifies whether it has reached the threshold value of number of nodes in the cluster. If the threshold has not been reached, it acknowledges the new node with $\mathrm{CH} \_\mathrm{ACK}$ (Table 2). The new node sends back JOIN (Table 2) with its node value. $\mathrm{CH}$ replies with $\mathrm{CH} \_\mathrm{NEWNODE}$ (Table 2) and broadcasts CH_INFO with updated neighbour node. Beyond threshold level, the $\mathrm{CH}$ replies with negative acknowledge $\mathrm{CH} \_\mathrm{NACK}$ (Table 2) to the new

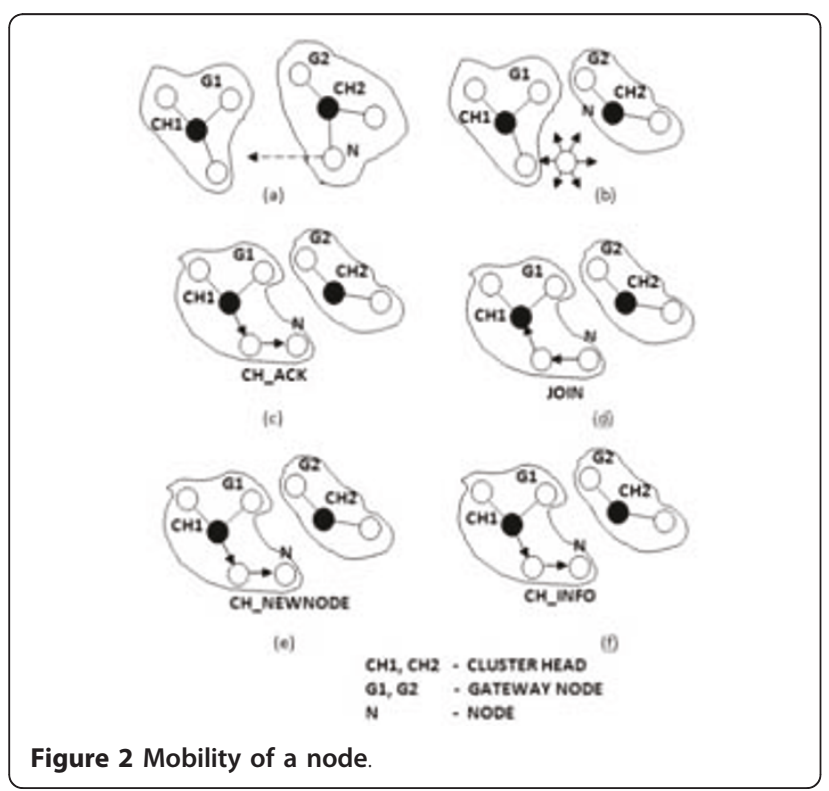

node. The new node repeats the above process with other CHs. It is explained in Figure 2.

\section{Mobility of Cluster Head}

When the $\mathrm{CH}$ moves away from the farthest node in the cluster, the farthest node waits for HELLO messages after a period of refresh time $T_{\text {ref. }}$. If the node receives the message, it still maintains the member state of the cluster. If it does not receive, it goes to undecided state. In the undecided state, it floods the neighboring node with HELLO message indicating its presence. Upon receiving the acknowledgement from any reachable $\mathrm{CH}$ or any other nodes in an m-hop cluster, it sends with its INFO message. Any reachable $\mathrm{CH}$ replies with its neighbor table and updates all the members in the cluster about the new node. The previous $\mathrm{CH}$ updates the neighbor table after every $T_{\text {ref }}$ and informs all the nodes.

\section{Route discovery}

The route discovery is done using source routing in cluster based routing protocols, whereas in ILCRP protocol it is done using location information. So control overhead becomes extremely high in cluster based routing protocols compared to location based routing protocols for source routing. Now, there are two instances of route discovery. The two instances are routing within a cluster known as intra cluster routing and routing between clusters known as inter cluster routing.

\section{Intra cluster routing}

In intra cluster routing, each and every node's GPS utility is made to sleep for reduced power consumption. All nodes in a cluster know about the location of other nodes in its cluster. Therefore, the source node forwards packets to the receiver node using the location information. If the destination node is one hop away from the receiver node, then source node sends the packet towards the destination node either using $\mathrm{CH}$ or using another node as shown in Figure 3. This process is explained in Algorithm 1.

Algorithm 1 as shown in Figure 3 is used for intra cluster routing in one hop cluster Node $S$ (source) checks its neighbour table for location information of Node D (destination)

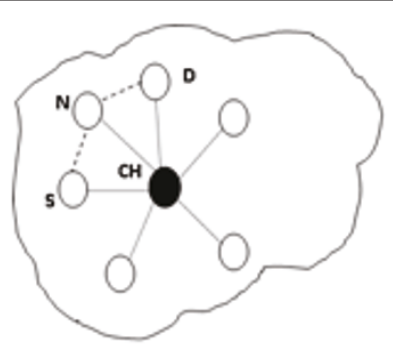

Figure 3 Intra cluster routing algorithm. 
Calculate the distance $\mathrm{D}_{\text {diff }}$ between the nodes having coordinates $S\left(x_{1}, y_{1}\right)$ and $D\left(x_{2}, y_{2}\right)$

$$
D_{\text {diff }}=\left|\sqrt{\left(x_{1}-x_{2}\right)^{2}+\left(y_{1}-y_{2}\right)^{2}}\right|
$$

If $\mathrm{D}_{\text {diff }}$ is greater than $\mathrm{D}_{\mathrm{txRg}}$ where $\mathrm{D}_{\mathrm{txRg}}$ is the maximum transmission range of the node.

Find one or more hop neighbours in the cluster

\section{If found}

Find the nearest neighbour node with less number of hops using the distance equation (2) and

Forward the packet to the node $N$ and

$N$ forwards the packet to Node D

$$
\begin{aligned}
& \text { Endif } \\
& \text { Endif } \\
& \text { Else }
\end{aligned}
$$

Node S forwards the packet towards Node D

\section{Endif}

When there is mobility of a node inside a cluster for a multi hop cluster, the use of LAR protocol results in higher efficiency. From Figure 4, Node D moves with an average speed of $v \mathrm{~m} / \mathrm{s}$ from known location at $t_{0}$. All the messages are routed to node $\mathrm{D}$ through $\mathrm{N} 1$ at $t_{0}$. After a time interval of $t_{\text {diff }}$, the node $\mathrm{D}$ is expected to be at a radius distance of $v t_{\text {diff }}$ units from the location at $t_{0}$. As shown in the Figure 4, Node D is not reachable via node N1. Using LAR, expected region is reachable via node N2. This process is explained in Algorithm 2.

Algorithm 2 as shown in Figure 4 is used for intra cluster routing in multihop $m(=2)$ cluster Follow the Algorithm 1 till the Node N1.

On receiving the packet, $N 1$ verifies whether the destination node is reachable

If (Not Reachable)
Find the estimated distance $R$ travelled by Node D in time $\Delta \mathrm{t}$

$$
R=v \Delta t
$$

Find the recent direction of node $D$ with deviation angle $\beta$ due to mobility $M$

The area of the circle shaped Request zone with radius $R$ is $\pi R^{2}$

Find the expected zone with same radius $R$ and deviation angle $\beta$.

Area of expected zone $=\frac{\beta}{360} \pi R^{2}$

Find the new node (N2) through which D is reachable Forward the packet through Node N2

N2 forwards the packet to Node D

Endif

Else

\section{Node N1 forwards the packet towards Node D}

\section{Endif}

The direction of destination node can be known by time differentiated GPS Coordinates (i.e., Direction, Latitude and Longitude). Therefore, the location of the destination node is identified and the beacon signal is transmitted within the expected zone by initially considering the value of $\beta=15^{\circ}$. If we are unable to catch up with the required destination node we increase the value of $\beta$ by $+/-10^{\circ}$. This procedure is repeated until the destination node is located.

\section{Inter cluster routing}

Using the CAT, the $\mathrm{CH}$ sends an inter-cluster Routing REQuest (RREQ) packet to its gateway nodes to obtain routing information between clusters in the form of source flooding. Routing REPly (RREP) Packet received from the destination contains the location information of the destination node, destination $\mathrm{CH}$, intermediate gateway node and source $\mathrm{CH}$.
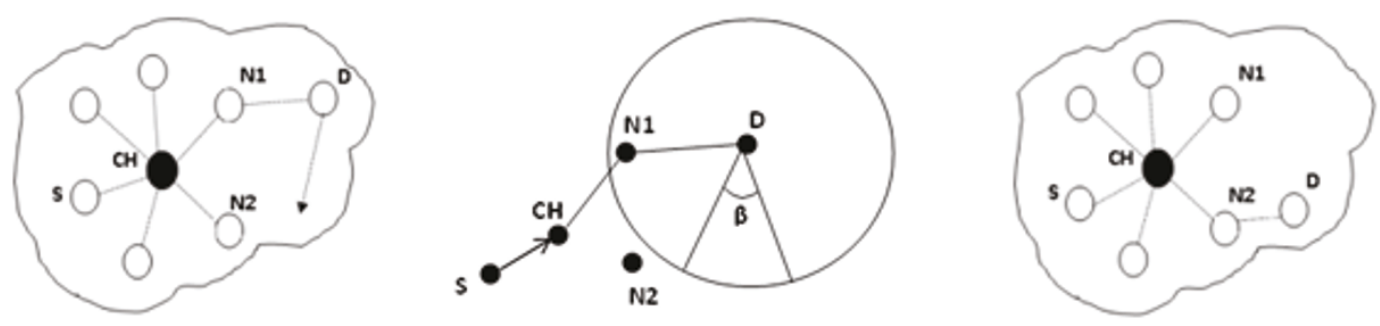

Figure 4 Intra cluster routing 
Consider the routing between adjacent clusters as shown in Figure 5. In a network of 2 clusters, routing is done using clusters as well as location information. Using the location information in RREP packet, the source node sends the packet directly towards the destination node through its gateway node. Gateway node forwards the packet to next cluster's gateway node. Gateway node calculates the expected and request zone for the destination node. If the expected zone does not fall in the transmission range of the gateway node, it forwards the packet to its $\mathrm{CH}$. Then the cluster forwards the packet to destination node through other nodes. This process is explained in Algorithm 3.

Algorithm 3 as shown in Figure 5 is used for inter cluster routing between adjacent clusters Source $S$ sends the RREQ (Route REQuest) packet to its CH (Cluster Head)

CH forwards the RREQ to adjacent cluster head via Gateway nodes $G$ in both the clusters.

On receiving the $R R E Q, C H$ checks its neighbour table and replies with RREP (Route REPly) packet containing the location information of destination Node D.

On receiving the location information of Node D, Node $S$ forwards the data packet to $G$ as per directional flooding.

After the data is received by the next cluster gateway node $G$, it calculates the expected zone as well as request zone as given in algorithm 2.

If Node $D$ is reachable

Node $G$ forwards the packet to the node D

Else if Node D is reachable via other nodes

Node G forwards the packet to Cluster Head of the destination node $D$

CH forwards the packet to Node D via other nodes in the cluster
Else

Node G replies NACK to Node $S$

Node $S$ requests the $\mathrm{CH}$ to reinitiate the route discovery process.

\section{End}

If the source cluster and destination clusters are $m$ clusters away, then the location information obtained by using initial source routing can be used for direction flooding. Consider the formation of clusters as shown in Figure 6, where Node S needs to send packet to Node D. Source $\mathrm{CH}$ forwards the packet using directional flooding with an angle of $\alpha$ via its gateway node. Now the packet hops from one cluster to another cluster by keeping closer to the axis of imaginary line between node $\mathrm{D}$ and source $\mathrm{CH}$. Transmission time of RREP from destination cluster $\mathrm{CH}$ to source $\mathrm{CH}$ is considered as $\Delta t_{1}$ whereas $\Delta t_{2}$ is the time taken by the packet to travel from source $\mathrm{CH}$ to the destination $\mathrm{CH}$.

Total time difference after finding the location information of the node is $D=\Delta t_{1}+\Delta t_{2}$. The velocity $(v)$ of the node $\mathrm{D}$ have already been obtained for calculation of the node value. This process is explained in Algorithm 4.

Algorithm 4 as shown in Figure 6 is used for inter cluster routing between clusters which are m clusters away After obtaining RREP, Node $S$ sends the packet to its $\mathrm{CH}$.

Source $\mathrm{CH}$ floods the packet directionally with an angle of $\alpha$ via its gateway Node.

After reaching the Destination $\mathrm{CH}$, it calculates the expected zone and request zone of the node $D$.

The request zone is given by the $\pi \mathrm{R}^{2}$ where

$$
R=v\left(\Delta t_{1}+\Delta t_{2}\right)=2 v \Delta t_{1} \quad \text { if } \quad \Delta t_{1}=\Delta t_{2}
$$

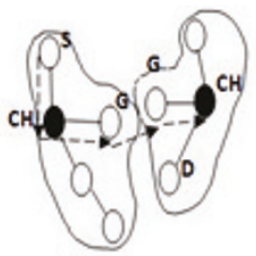

(a)

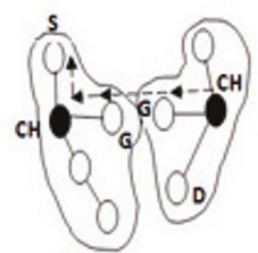

(b)
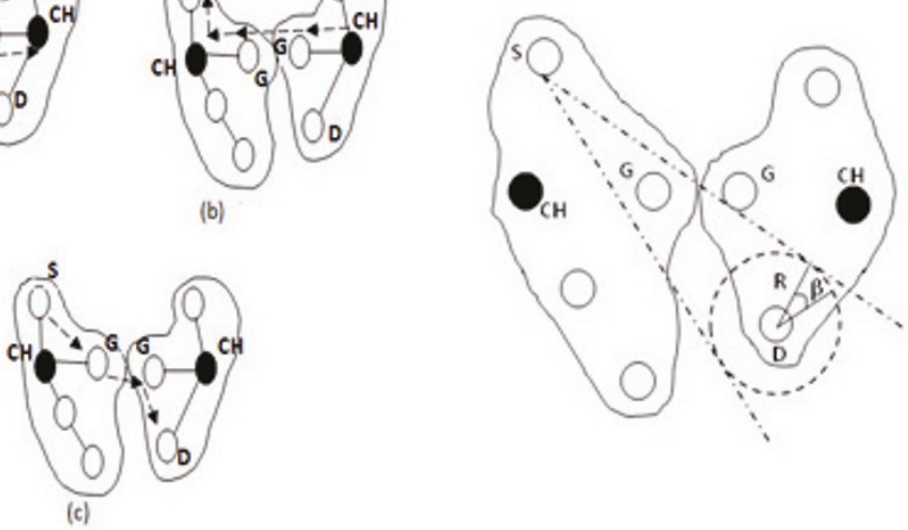

Figure 5 Inter cluster routing. (a) Flow of RREQ. (b) Flow of RREP. (c) Flow of data. (d) Intercluster routing between adjacent clusters. 


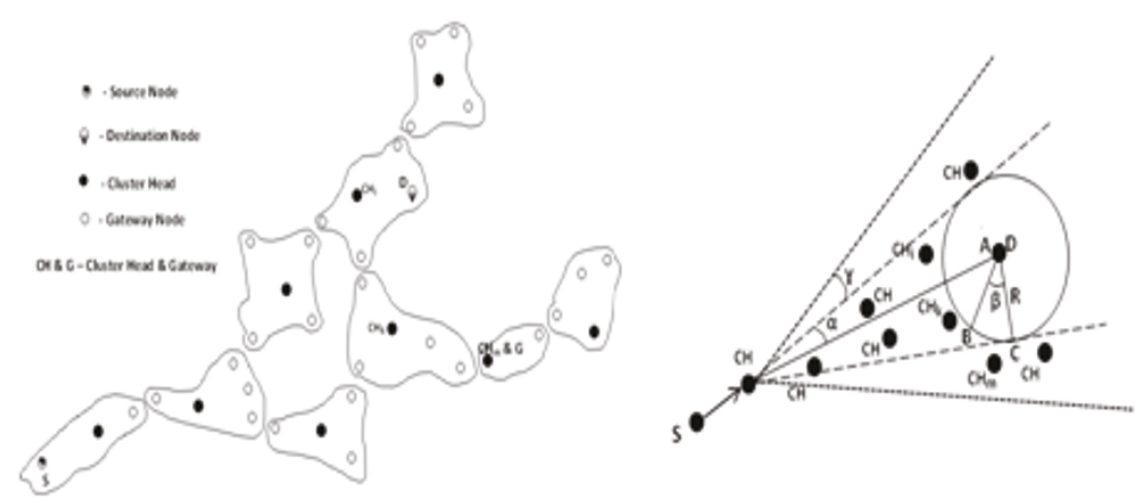

Figure 6 Inter cluster routing between clusters which are $m$ clusters away

As the direction of the node $D$ is known, Area of the expected zone is calculated by

$$
\frac{\beta}{360} \pi\left(2 v \Delta t_{1}\right)^{2}
$$

If Node D is present in the cluster

CH forwards the packet to Node D

Else

CH forwards the packets directionally to the clusters

\section{End}

\section{Route recovery}

If a route failure occurs due to movement of the nodes in the intermediate clusters, the path should be reinitiated either from the local node where route failure is detected or from the source $\mathrm{CH}$. Initially the path rediscovery starts from the local node by directional flooding. If the local rediscovery fails, the local nodes inform the source $\mathrm{CH}$. The source $\mathrm{CH}$ increases the directional flooding angle $\alpha$ by $\gamma$ as shown in Figure 6 .

\section{Simulation results}

\section{Simulation parameters}

- Performed using NS-2 network simulator [30] with MANET extensions.

- IEEE 802.11 is used as the MAC layer protocol.

- The radio model simulates with a nominal bit rate of 2 Mbps.

- Nominal transmission range is $125 \mathrm{~m}$.

- The radio propagation model is the two-ray ground model.

- First 100 nodes are deployed for one experiment and then 100 nodes are used for another experiment in a field of $1000 \mathrm{~m} \times 1000 \mathrm{~m}$.

- The traffic pattern is CBR (constant bit rate) with a network traffic load of 4 packet/s and the packet length are 512 bytes.
- The mobility model used is the Random Waypoint Model

- The pause time of the node reflects the degree of the node mobility. The small pause time means intense node mobility and large pause time means slow node mobility. The pause time is maintained as $5 \mathrm{~s}$.

- The simulation time is $900 \mathrm{~s}$.

- The first set of simulations are performed by varying the speed from 2 to $10 \mathrm{~m} / \mathrm{s}$ with an increment of $2 \mathrm{~m} / \mathrm{s}$ keeping number of nodes constant to 40 .

- The second set of simulations are performed by creating 20,40,60, 80, 100 nodes, keeping speed constant to $5 \mathrm{~m} / \mathrm{s}$.

- The value of weights $\mathrm{W}_{1}, \mathrm{~W}_{2} \mathrm{~W}_{3}$, for simulation are $(0.09,0.38,0.53),(0.27,0.31,0.42)$ and $(0.33,0.24,0.43)$, respectively.

\section{Performance metrics}

For evaluating the performance of ILCRP, the metrics chosen are packet delivery ratio, control overhead and end to end delay.

\section{End to end delay}

End to end delay indicates the time lapse between the source and destination nodes in the network. Figures 7 and 8 shows that the end to end delay reduces if the exact locations of all the nodes are obtained. On increasing the mobility of the nodes, the delay increases due to reconfiguration of the clusters. The end to end delay also increases due to increase in the number of nodes due to more number of hops.

\section{Packet delivery ratio}

It is defined as the ratio of total number of packets that have reached the destination node to the total number of packets originated at the source node. The location information of the nodes make the packets route, loop free which results in high packet delivery ratio. On increasing the mobility or speed of the nodes, the delivery ratio decreases since most of the nodes move away 


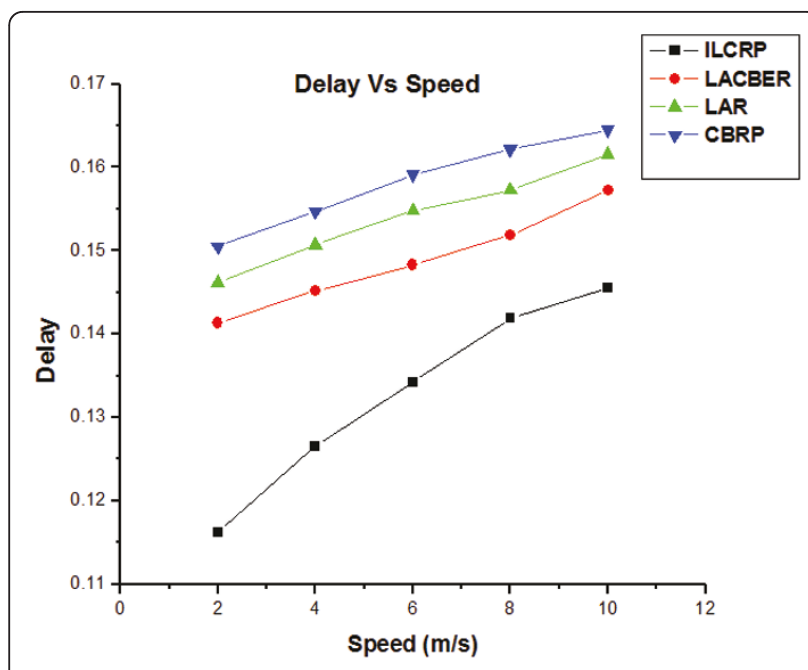

Figure 7 Comparison for delay vs. speed.

from each other. Increasing the number of nodes decreases the delivery ratio due to tightly coupled cluster configuration. Figures 9 and 10 confirms the packet delivery ratio between ILCRP and LACBER, LAR, CBRP. Control overhead

It is defined as the ratio of the number of control packets transmitted to the number of the data packets delivered. Usage of cluster based routing protocol for clustering and exact location information for route discovery reduces the control overhead in the network. Figures 11 and 12 shows the control overhead ratio between ILCRP, LACBER, LAR and CBRP. It increases when the mobility of the nodes as well as number of nodes increases.

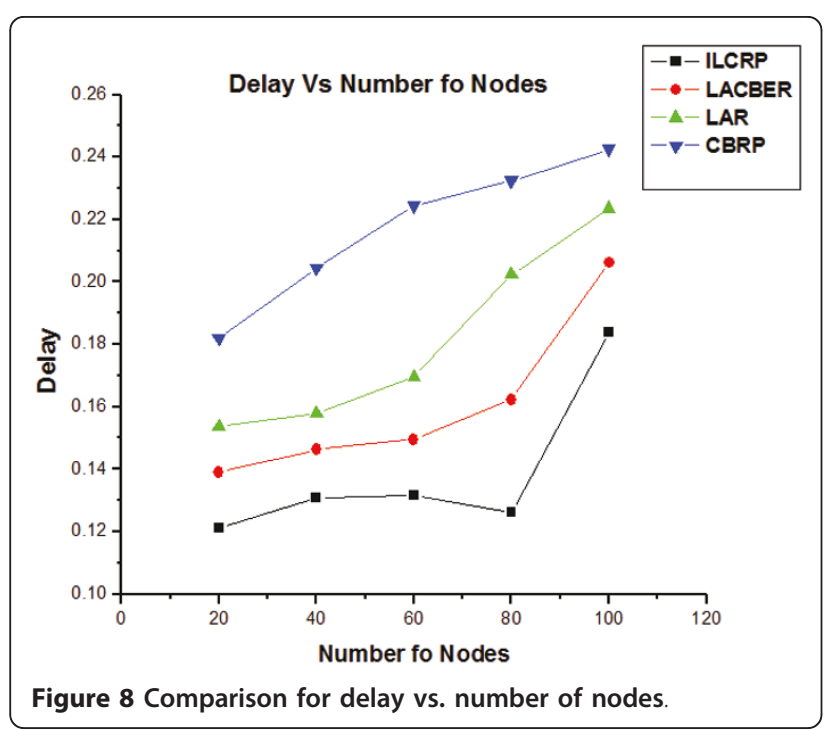

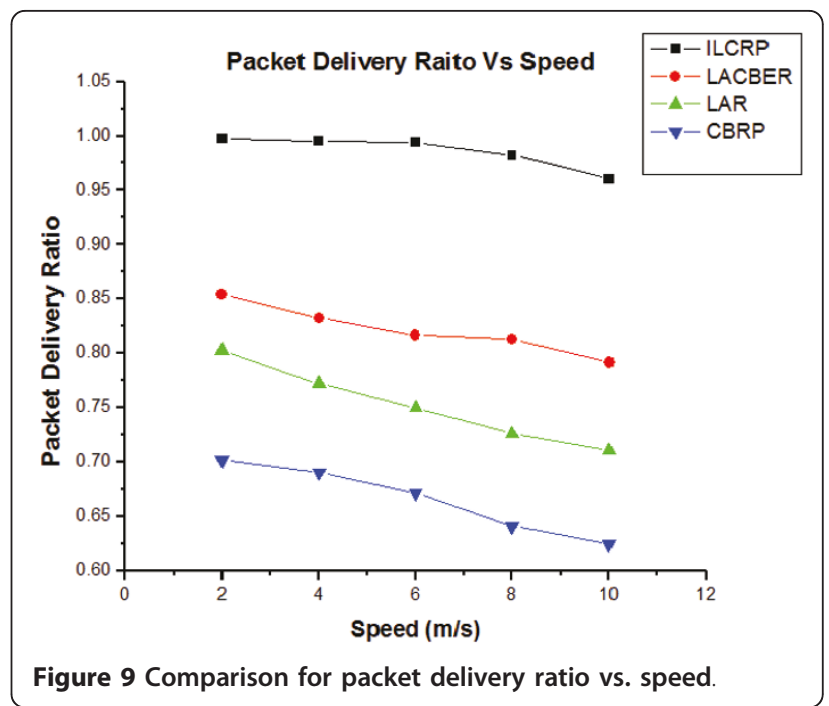

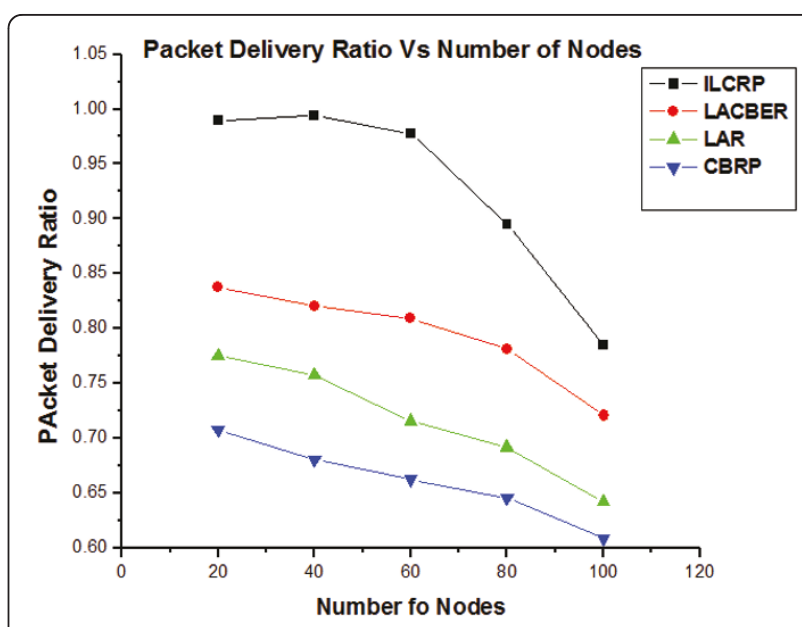

Figure 10 Comparison for packet delivery ratio vs. number of nodes.

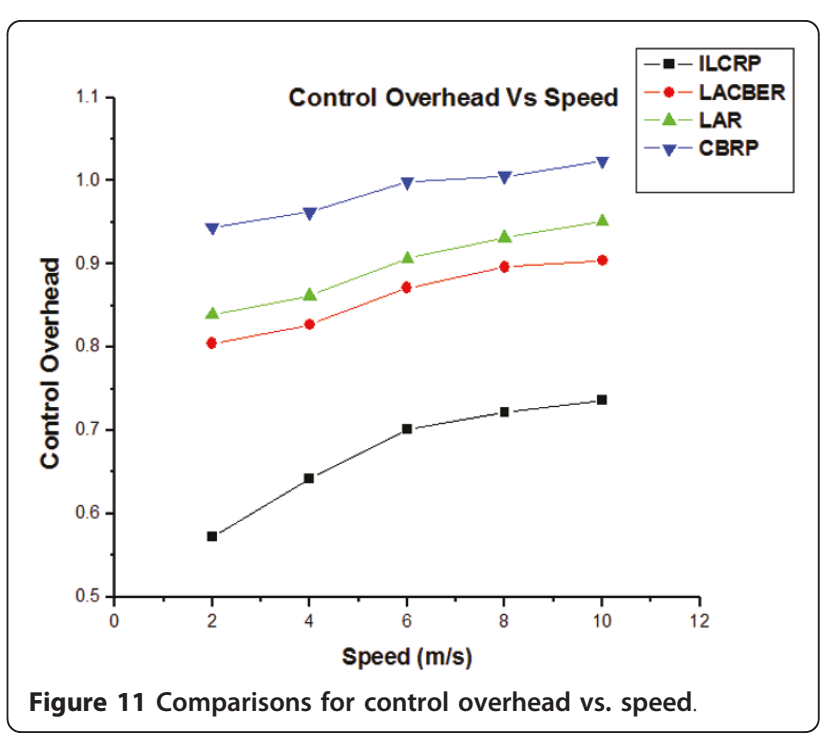




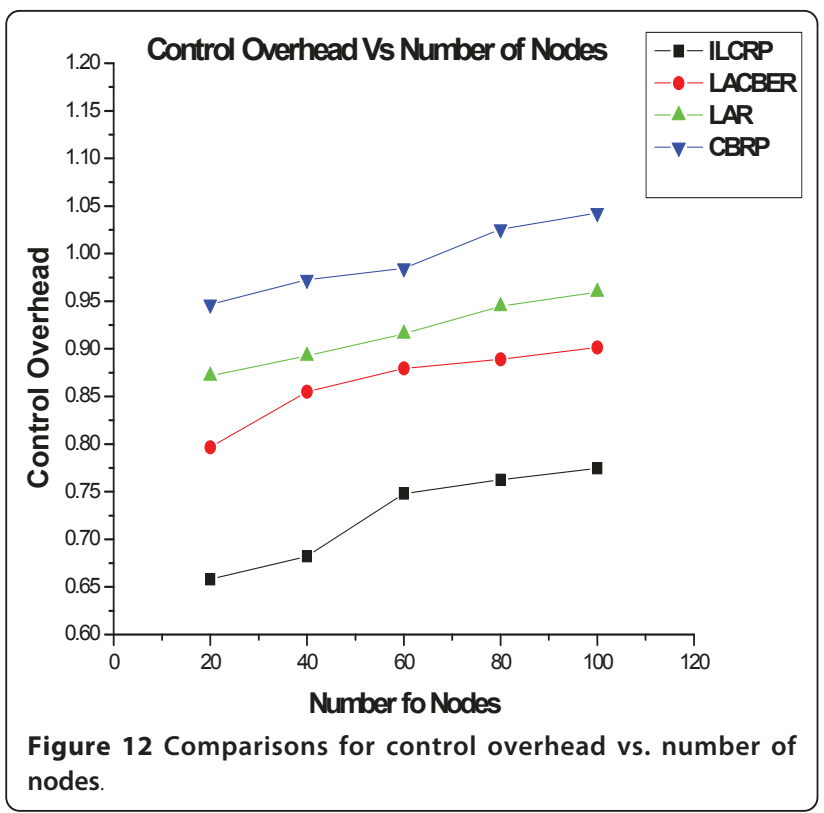

\section{Conclusion}

This paper introduces a new stable clustering scheme that are applicable in highly mobile ad hoc networks. Use of location information in the $\mathrm{m}$-hop cluster based routing forms the basis of ILCRP. The exact location information of nodes in ILCRP increases the delivery ratio and reduces the control overhead and makes the route, loop free. Location information of all the nodes keeps the exchange information as well as the end to end delay very low in ILCRP compared to other protocols. From the results, it can be seen that the proposed scheme performs better than GPS free as well as GPS Scarce MANETs as the proposed scheme forms stable clusters containing members that remain within their associated clusters for a longer period of time, despite the targeted system having node speeds exceeding normal MANET scenarios. It is hoped that the geographic routing based clustering scheme presented would form the foundation for the possibility of reliable data sharing and communication between highly mobile vehicles i.e., VANETs for the present and in the future.

\section{List of Abbreviations}

AOA: angle of arrival; CAT: Cluster Adjacency Table; CBRP: Cluster Based Routing Protocol; $\mathrm{CH}$ : cluster head; DMAC: Distributed Mobility Adaptive Algorithm; GDMAC: Generalized Distributed Mobility Adaptive Clustering; HD: Highest-degree; ILCRP: Improved Location aided Cluster based Routing Protocol; LAR: Location Aided Routing; LACBER: Location Aided Cluster Based Energy-efficient Routing; MOBIC: Mobility Metric Based Algorithm; RREP: Routing REPly; RREQ: Routing REQuest; WCA: Weighted Clustering Algorithm.

\section{Author details}

${ }^{1}$ Department of Electronics \& Communication Engineering, Velalar College of Engineering and Technology, Thindal, Erode-638 012, Tamil Nadu, India
${ }^{2}$ Department of Computer Science and Engineering, Kongu Engineering College, Perundurai-638 052, Tamil Nadu, India

\section{Competing interests}

The authors declare that they have no competing interests.

Received: 23 September 2010 Accepted: 17 June 2011 Published: 17 June 2011

\section{References}

1. A Iwata, C Chiang, G Pei, M Gerla, T Chen, Scalable routing strategies for ad hoc wireless networks. IEEE J Select Areas Commun. 17(8), 1369-1379 (1999)

2. A Ephremides, JE Wieselthier, DJ Baker, A design concept for reliable mobile radio networks with frequency hoping signaling. Proc IEEE. 75(1), 56-73 (1987)

3. M Gerla, JTC Tsai, Multicluster, mobile, multimedia radio network. Wireless Netw. 1, 255-265 (1995)

4. AK Parekh, Selecting routers in ad hoc wireless network. in Proceedings of the SBT/IEEE International Telecommunication Symposium, ITS, August 1994

5. P Basu, N Khan, TDC Little, A mobility based metric for clustering in mobile ad hoc networks. in Proceedings of IEEE ICDCS 2001 Workshop on Wireless Networks and Mobile Computing, Phoenix, AZ, 2001

6. S Basagni, Distributed clustering for ad hoc networks. in Proceedings of International Symposium on Parallel Architectures, Algorithms and Networks, June 1999, pp. 310-315

7. S Das, M Chatterjee, D Turgut, WCA: a weighted clustering algorithm for mobile ad hoc networks. J Cluster Comput. 5(2), 193-204 (2002)

8. S Das, M Chatterjee, D Turgut, An on demand weighted clustering algorithm (WCA) for ad hoc networks. in Proceedings of IEEE GLOBECOM 2000, San Francisco, November 2000, pp. 1697-1701

9. M Chatterjee, SK Das, D Turgut, A weight based distributed clustering algorithm for mobile ad hoc networks. in Proceedings of 7 th International Conference on High Performance Computing (HiPC 2000), Bangalore, India, December 2000, pp. 511-521

10. R Ghosh, S Basagni, Limiting the impact of mobility on ad hoc clustering. in Proceedings of the 2nd ACM International Workshop PE-WASUN'05, Montreal, CA, 2005, pp. 197-204

11. X Zhao, X Gu, Z Sun, C Ren, An intelligent weighted clustering algorithm (IWCA) for ad hoc. in Software Engineering (WCSE '09), 2009

12. Z El Bazzal, M Kadoch, BL Agba, F Gagnon, M Bennani, A flexible weight based clustering algorithm in mobile ad hoc networks. in International Conference on Systems and Networks Communication (ICSNC'06), Tahiti, French Polynesia, 2006

13. J Sucec, I Marsic, Clustering overhead for hierarchical routing in mobile ad hoc networks. in IEEE Infocom, 2002

14. M Jiang, J Li, YC Tay, CBRP-IETF MANET Draft. National University of Singapore, August 1999

15. Z Chunhua, T Cheng, A K-Hop passive cluster based routing protocol for MANET. in 5th International Conference on Wireless Communications, Networking and Mobile Computing, WiCom'09, 2009

16. YB Ko, NH Vaidya, Location-aided routing (LAR) in mobile ad hoc networks. in Proceedings of the 4th Annual ACM/IEEE International Conference on Mobile Computing and Networking, 25-30 October 1998,. pp. 66-75

17. BW Parkinson, JJ Spilker, Global positioning system: theory and application. Prog Astronaut Aeronaut. 1, 163 (1996)

18. M Mauve, J Widmer, $\mathrm{H}$ Hartenstein, A survey on position-based routing in mobile ad hoc networks. IEEE Netw Mag. 15, 30-39 (2001)

19. GS Tomar, RS Tomar, Position based routing for mobile ad hoc networks. in Second UKSIM European Symposium on Computer Modeling and Simulation, pp. 555-560 (2008)

20. MA Mikki, Energy efficient location aided routing protocol for wireless MANETs. Int J Comput Sci Inform Security 4,(1-2) (2009)

21. S Capkun, M Hamdi, JP Hubaux, GPS free positioning in mobile ad hoc networks. in Cluster Comput J. 5(2), 157-167 (2002)

22. A Caruso, S Chessa, S De, R Urpi, GPS free coordinate assignment and routing in wireless sensor networks. in Proceedings of the IEEE INFOCOM, 2005, 24th Annual Joint Conference of the IEEE Computer and Communications Societies, pp. 150-160 (2005) 
23. H Akcan, V Kriakov, N Bronnimann, GPS-Free node localization in mobile sensor networks. in Proceedings of the 5th ACM International Workshop on Data Engineering for Wireless and Mobile Access, pp. 35-42 (2006)

24. R lyenger, B Sikdar, Scalable and distributed GPS free positioning for sensor networks. in Proceedings of IEEE Conference on Communication ICC. 2003, vol. 1, pp. 338-342

25. OH Kwon, HJ Song, Counting-Based Distance Estimations and Localizations inWireless Sensor Networks. Springer., 2006: 3983/2006, pp. 306-315

26. HC Chu, RH Jan, A GPS-less, outdoor, self positioning method for wireless sensor networks. Ad Hoc Netw (Elsevier Sci). 5(5), 547-555 (2007). doi:10.1016/j.adhoc.2006.03.004

27. N Bulusu, J Heidemann, D Estrin, GPS-less low cost outdoor localization for very small devices. in IEEE Personal Communications Magazine, Special Issue on Smart Spaces and Environments, October 2000

28. D Deb, SB Roy, N Chaki, LACBER: a new location aided routing protocol for GPS scarce MANET. Int I Wireless Mobile Netw (IJWMN), 1(1), 22-36 (2009)

29. SK Jahanbakhsh, M Hajhosseini, Improving performance of cluster based routing protocol using cross-layer design arXiv.org>cs>arXiv:0802.0543v1, pp. $731-742$

30. The Network Simulator ns-2. (Information Sciences Institute, USA. Viterbi School of Engineering, September, 2004) Available: http://www.isi.edu/ nsnam/ns/

doi:10.1186/1687-1499-2011-18

Cite this article as: Mangai and Tamilarasi: A new approach to

geographic routing for location aided cluster based MANETs. EURASIP

Journal on Wireless Communications and Networking 2011 2011:18.

\section{Submit your manuscript to a SpringerOpen ${ }^{\circ}$ journal and benefit from:}

- Convenient online submission

- Rigorous peer review

- Immediate publication on acceptance

- Open access: articles freely available online

- High visibility within the field

- Retaining the copyright to your article

Submit your next manuscript at $\gg$ springeropen.com 